\title{
La universidad católica: desafíos y promesas ${ }^{1}$
}

\section{Monseñor Guy-Réal Thivierge ${ }^{\star}$}

\section{Introducción}

Intentaré insertarme en el tema elegido para esta reflexión, tomando las ideas que deseo compartir con ustedes, primero, de las fuentes de mi experiencia universitaria personal, y, en segundo lugar de las fuentes de la Federación Internacional de Universidades Católicas (FIUC), observatorio privilegiado de sensibilidades, cuestionamientos y prácticas universitarias del mundo.

Iniciaré tratando de elaborar una panorámica de algunos desafíos a los que están llamadas a responder las universidades católicas hoy. Que marcan cada vez más la identidad de nuestras instituciones, nuestra manera de vivir la misión, sin olvidar la calidad de nuestras interacciones, y hasta de nuestras cooperaciones. De ahí el título de mi reflexión: La universidad católica: desafios y promesas.

Parto de una constatación: la universidad se encuentra en una fase de transformación importante bajo la presión de exigencias diversas. Ya sea del Norte o del Sur, rica o pobre, privada o pública, comparte fundamentalmente las mismas preocupaciones, se plantea cuestiones sobre su razón de ser y su desarrollo futuro. Elabora su balance y reevalúa sus misiones frente a los desafíos que le imponen las sociedades actuales, en el plano local y en el global. De una forma u otra, parece haber una cuestión en el corazón de la reflexión universitaria: ¿cómo poner la ciencia y la investigación científica, las artes y sus prácticas, los saberes, los conocimientos y las técnicas, productos de la inteligencia y de la imaginación del género humano, al servicio de lo humano, subentendido de una manera absolutamente particular en la universidad católica, de todo lo humano? ¿Cómo conseguirlo, habida cuenta de las solicitaciones que nos apremian por todas partes, desde dentro y desde fuera, a la eficiencia y a la eficacia? ¿Cómo resistir, en un contexto de globalización, a la ofensiva de los mercados? ¿Se ha convertido el conocimiento en un producto de consumo que se pueda comprar o vender? Aquí tenemos un primer conjunto de cuestiones que se han vuelto

\footnotetext{
1 Ponencia presentada en el VI Encuentro de Reflexión Universitaria de la Universidad Politécnica Salesiana, 27 de junio de 2008, Cuenca-Ecuador.

Secretario General de la Federación Internacional de Universidades Católicas (FIUC). E-mail: sgfive@bureau.fic.org
} 
ineludibles hoy en las universidades, públicas o católicas. ¿Cómo marcan estos desafíos y las apuestas que implican el presente y el futuro de las instituciones? ¿De qué recursos dispone la universidad católica para responder a estos interrogantes? ¿Cómo puede seguir siendo fiel a la magna tradición universitaria a la que pertenece y, al mismo tiempo, abrirse con sabiduría a los imperativos de la posmodernidad?

Ya lo habrán presentido, la empresa es inmensa, compleja, y supera ampliamente el marco de una intervención como ésta. Voy a esbozar algunas facetas de la puesta en tela de juicio a la universidad de hoy y procuraré examinar, de un modo más específico, algunos de los desafíos que debe enfrentar: el desafío del desarrollo científico y tecnológico, el de la implantación de una sociedad del conocimiento, la ofensiva de los mercados sobre la universidad, la internacionalización de la educación superior "vía Internet".

\section{La Universidad en tela de juicio}

Es poco decir que el sentido y la misión de la universidad, institución plurisecular procedente de la Edad Media y reformada, por vez primera, al alba de la Revolución científica y técnica del siglo XIX, haya perdido una parte de su evidencia en nuestras sociedades. Los cuestionamientos de la universidad, privada o pública, sobre sí misma resultan tanto de tendencias a largo plazo (la vieja cuestión de la masificación, la adaptación de los sistemas educativos a las evoluciones de los distintos medios) como de mutaciones más recientes (la globalización económica y el papel crucial que en ella desempeña la innovación tecnológica, la búsqueda de flexibilidad en la oferta educativa, el abandono del papel centralizador del Estado, y hasta su falta de implicación, el desarrollo de lo que se llama -permítanme la expresión-, la business education, entre otras).

Asimismo, es verdad que el paradigma conocido de la organización de los saberes se encuentra también en dificultades, y el enfoque clásico de la reproducción cultural y de la difusión del saber ha sido puesto en tela de juicio. Por un lado, si bien es evidente que el saber y el conocimiento son en nuestros días una exigencia social para el desarrollo y el bienestar de las sociedades; también es cierto que eso mismo engendra, no solo una demanda creciente de formación superior, sino de igual forma, la necesidad de una cooperación reforzada entre 
las diferentes disciplinas, los diferentes centros de producción de cultura y de conocimiento, los diferentes saberes (humanista, científico, técnico). La enseñanza ya no puede organizarse siguiendo el principio del aprendizaje pasivo de unas nociones establecidas, sino en función de la necesidad de aprender a enseñar de manera global y contextual. Por otro lado, la hiperespecialización y la compartimentación disciplinaria impiden con excesiva frecuencia el acceso a los conocimientos principales, a la cultura general.

Además, se pide de la misma forma a la universidad que se someta a los mercados, so pretexto de garantizar la salida profesional de sus estudiantes. Esta relación universidad-mercado (universidad-empresa) no dominada, corre el riesgo de transformar la universidad en "colegio superior de formación profesional" y en una empresa económica entre otras. La universidad se arriesga así a perder su autonomía, a ver subordinar lo académico a lo económico, y a pasar a segundo plano la enseñanza superior (en el sentido amplio del término) y con ella la libertad académica, la investigación y la difusión de la cultura.

La universidad, pública o católica, debe preservar su alma defendiendo el principio de la universitas, el principio que la ha venido inspirando desde su nacimiento, como lugar autónomo de reflexión, de investigación y de difusión de los saberes. Un ámbito donde las diversas aproximaciones al conocimiento y las diferentes culturas, sin otro fin que la búsqueda de la verdad, puedan dialogar libremente y al margen de toda lógica de pura rentabilidad económica, y sustrayéndose a cualquier coerción de orden nacional, económica o política.

De acuerdo con sus funciones, que incluyen hoy no solo la investigación, la enseñanza (formación) y el servicio a las colectividades, aunque también la apertura y la cooperación internacional, la universidad debe seguir siendo un ambiente prioritario para conjugar pensamiento, saber, enseñanza, formación, reflexión y educación. Un espacio donde se mezclen las culturas, las ideas, las lenguas... Un ámbito capaz de dar a la inteligencia de lo público -sea cual sea- posibilidades de aprender a mirar, escuchar, apreciar, criticar, evaluar las ciencias y las técnicas, la calidad de un texto, de una representación escénica, de una interpretación, de una obra artística. Éstos son los instrumentos necesarios para que cada ser humano pueda construir su propia visión del mundo, y compartir, en medio de la diferencia y la pluralidad, la de los otros, siempre con la mirada puesta en la construcción de un destino común, y ¿por qué no? una 
casa común. ¿Acaso no tiene también la universidad como vocación contribuir a remediar el déficit de sentido que parece afectar al conjunto de sectores que forman la sociedad? ¿No sigue estando encargada de transmitir una cultura fundadora del bien común, de la democracia y de la esperanza?

En virtud de su papel de difusión de la cultura y del saber, la universidad se encuentra en el corazón, no solo de la investigación, sino también de la voluntad permanente de inteligibilidad. Nosotros sabemos que las dos problemáticas de la interpretación del mundo y de su transformación son solidarias. Sabemos también que si la transformación del mundo se presenta como asunto de todos, el dominio de esta transformación sigue siendo aún un campo reservado a una minoría. El saber y el conocimiento científico son depositarios de un poder que no es ni neutro ni objetivo. Actúa a favor o en contra de la vida y del bienestar. La universidad y los universitarios tienen, por tanto, la responsabilidad ética y ciudadana de la obra científica y técnica.

Es necesario que haya un ámbito como la universidad, que asuma no solo la responsabilidad ética y civil de la ciencia, sino que participe en la ciudadanía y en el buen vivir juntos mediante la promoción de la cultura. La cultura que permite conocerse, pues ella es vector de identidad, y reconocerse, porque es capaz de reconocimiento, sin el cual nace la división, la marginación. La cultura es un patrimonio colectivo y resulta determinante en nuestra relación con el otro, en nuestra respuesta a la llamada del otro, en el espacio público y privado. Es también un remedio para la desconfianza y el odio. La invitación al diálogo entre las culturas y las religiones debe resonar también en la universidad como una invitación al aprendizaje, a la formación para el diálogo, y encontrar así, en nuestros medios, proposiciones coherentes, estructuradas y pedagógicas capaces de ayudar, en sus misiones respectivas, tanto a la sociedad civil como a la Iglesia.

\section{El desafío del desarrollo científico y tecnológico}

La universidad en general tiene la responsabilidad ética y ciudadana de la obra científica y técnica. En efecto, la universidad se enfrenta hoy a ciertos desafíos, efecto de los progresos que ha realizado, los mismos que han adquirido dimensiones planetarias. El desarrollo económico de una gran cantidad de países, ligado sin la menor duda al progreso de los conocimientos técnico- 
científicos, ha hecho a la humanidad consciente de su fuerza y, por ende, también de su capacidad para enfrentar con éxito el problema del hambre y de las enfermedades que la han atormentado durante siglos. Lo que parecía ayer un problema insuperable, casi una imposibilidad, aparece hoy posible y realizable, desde el punto de vista puramente técnico. Con todo, muchos países viven todavía en la indigencia y en el subdesarrollo. "Este mismo hombre, escribe el Papa Juan Pablo II, que es el artífice de tantas nuevas posibilidades, es también, con excesiva frecuencia, espectador de muchas imposibilidades prácticas, cuando no responsable directo de los impedimentos que se oponen a la extensión del desarrollo y de sus beneficios". ${ }^{3}$ Y qué decir del mismo desarrollo cuando es comprendido de una manera exclusivamente económica y unilateral.

Este contraste debe ser iluminado y curado de una manera perspicaz. Tiene su origen en la voluntad del hombre y no será superado más que gracias a un esfuerzo moral intenso y renovado, que tendrá su fuente en la reflexión sobre el misterio del hombre, un ser capaz de grandeza y de miseria, y sobre el verdadero fundamento transcendente de la justicia. El desarrollo técnico-científico conduce, junto a innegables ventajas para la humanidad, a aspectos problemáticos e inquietantes, que requieren también una gran profundización ética responsable. Pensemos, por ejemplo, en la crisis de tantas ideologías y modelos de conducta que se suceden sobre el escenario cambiante de nuestro tiempo y que dejan siempre a muchas personas, poblaciones y sociedades en una situación de falta de identidad y de conmoción existencial. Más cerca de nosotros, la caída del muro de Berlín, llena de esperanzas y de promesas de libertad, ha traído también consigo nuevas alienaciones de las que somos testigos en la vida diaria en Europa.

Estos desafíos se dirigen también a la universidad, en cuanto tal, los encontramos en los medios en que vivimos, en que trabajamos, y son comunes a todas las universidades. Los hallamos también en el ámbito de cada nación y están presentes en los grandes debates de organismos internacionales como la UNESCO y el Consejo de Europa. Se han indicado caminos, se han propuesto soluciones ricas en elementos pertinentes y estimulantes. Los análisis

3 Juan Pablo II, Discurso a los participantes en el Congreso internacional de las Universidades católicas y de los Institutos de Estudios Superiores (25 de abril de 1989), 2. El texto italiano fue publicado en "l'Osservatore Romano" del 25 de abril de 1989. 
profundos ponen de relieve que no podemos buscar las respuestas solo en el ámbito social, como si bastara con acercar la universidad a las preocupaciones y necesidades de la sociedad, y convertir a ésta en el lugar de preparación de una fuerza de trabajo eficaz destinada al buen funcionamiento del aparato de producción. Las respuestas tampoco pueden reducirse a realizar un mayor esfuerzo en el plano de la organización académica, multiplicando las universidades, los institutos especializados, las facultades y los departamentos. Eso puede ser necesario, es cierto, pero nunca será suficiente, pues los desafíos tienen que ver con cuestiones de fondo. Lo que está en juego es el sentido mismo de la investigación científica y de la tecnología, de la convivencia social, de la cultura, y, yendo más al fondo, es la significación misma del ser humano lo que está en cuestión. Dicho con otras palabras, y adoptando un punto de vista más general, podríamos decir que estos desafíos tienen que ver con la verdad sobre el hombre en su dimensión personal y social; con la verdad sobre el mundo con sus leyes, que debemos descubrir y emplear para el bien de la humanidad; con la verdad sobre Dios, el Ser fundador, a quien todo debe ser reconducido y que es el único que da su significación última al hombre y al mundo. ${ }^{4}$

\section{El desafío de la sociedad del conocimiento}

En el entorno globalizador que conocemos, todas las economías, pero de modo especial las llamadas economías en desarrollo o en transición, deben hacer frente a nuevos desafíos que afectan no solo a los modelos y las prácticas de la misma economía, sino que trastornan los objetivos (y las prácticas) de la educación superior. Entre los aspectos más críticos que traen consigo estos cambios es preciso señalar, en primer lugar, el creciente papel del conocimiento como factor de crecimiento económico, $\mathrm{y}$, a continuación, el lugar central de la revolución ligada a las nuevas tecnologías de la información y de la comunicación. La producción y el acceso al conocimiento, así como a las aplicaciones que de él se desprenden, se han convertido en factores determinantes del desa-

\footnotetext{
4 Chesterton ha escrito: "nadie puede comprender la filosofía... católica, sin comprender primero que ésta es, fundamentalmente, elogio de la vida, elogio del ser, elogio de Dios como creador del mundo" (G.K. Chesterton, Santo Tomás de Aquino, Espasa-Calpe, Madrid 1948, p. 94).
} 
rrollo económico y se encuentran cada vez más en el corazón de la dinámica de competición que los países deseosos de tomar parte en el mercado global han puesto por delante. La combinación de los progresos informáticos, la disminución de sus costes de producción, los avances de las tecnologías sin cable y vía satélite, al igual que la reducción del precio de las telecomunicaciones, todo ello junto ha permitido levantar las barreras del tiempo y del espacio que otrora obstaculizaban el acceso a la información y al intercambio.

Estos cambios provocan el surgimiento de nuevas posibilidades y nuevos desafíos. Su aspecto positivo es que el papel de la educación superior en la construcción de las economías del conocimiento y de las sociedades democráticas se vuelve más influyente que nunca. En efecto, la educación superior se encuentra en el corazón de la formación intelectual de las que dependen la producción de los conocimientos, su aplicación y su diseminación, especialmente en lo que respecta a la formación individual y profesional, así como por la que se dispensa a lo largo de toda la vida. Se manifiesta otro desarrollo favorable a través de la aparición de nuevos tipos de instituciones de enseñanza superior y nuevas formas de competición, que incitan así a las instituciones tradicionales a adaptar sus modos de funcionamiento y de presencia ante los medios a los que desean servir. Por ejemplo, la educación a distancia, accesible con frecuencia a los menos favorecidos, se ha consolidado sobremanera con el advenimiento de las nuevas tecnologías de la información y de la comunicación. Su aspecto negativo es que estas transformaciones engendran el peligro de lo que hoy llamamos la fractura digital en el seno de las naciones y entre unas y otras. Las mayorías excluidas se vuelven todavía más pobres y las elites globalizadas se hacen cada vez más ricas.

Aunque nacen nuevas posibilidades para la educación superior, la mayoría de los países en vías de desarrollo o en proceso de reconstrucción continúan luchando por el mantenimiento, si no por la supervivencia, de una educación superior de calidad y capaz de incidir realmente sobre las posibilidades y las numerosas expectativas locales. Entre éstas mencionaremos la fragilidad de los sistemas públicos de educación y la fuerte demanda de compensación que pesa sobre la enseñanza privada, en nuestro caso frecuentemente sobre las universidades católicas. A eso se debe añadir la inestabilidad política y la falta de 
recursos profesionales y financieros, que debilitan a menudo la administración y la gestión de las instituciones y comprometen su estabilidad (durabilidad).

Otros aspectos ligados a las políticas del Banco Mundial como: la reducción de la pobreza a través del crecimiento económico, la redistribución de capitales y la formación de todo tipo para el servicio de la producción, contribuyen también a reducir el conocimiento a un bien de consumo accesible a los más favorecidos económicamente.

\section{El desafío de los mercados sobre la Universidad}

¿Qué entendemos por la ofensiva de los mercados sobre la universidad? Dicho de otro modo, ¿cuáles son los ejes esenciales de la "mercantilización” de la enseñanza superior? Tal como yo decía más arriba, bajo la presión de un entorno económico cada vez más competitivo e imprevisible, de un mercado del trabajo polarizado, de dificultades presupuestarias crecientes ( $¡ y$ bien sabe Dios cómo se enfrentan a esta dificultad las universidades católicas!) y de mercados financieros en busca de inversiones rentables, los sistemas de enseñanza superior tienden a evolucionar hacia una mayor diferenciación, hacia una mayor flexibilidad, hacia una mayor instrumentalización al servicio de la competición económica $\mathrm{y}$, como último punto de convergencia de estas tres tendencias, hacia la privatización mercantil. El conjunto de este proceso se encuentra favorecido además por la difusión de una potente ideología, neoliberal, que es, a la vez, producto y condición ideológica necesaria del inmenso movimiento de globalización mercantil que afecta ahora a todos los aspectos de la vida de los hombres y de las mujeres de hoy.

Encontramos un ejemplo de lo que decimos en esta profesión de fe de un responsable del Banco Mundial:

La competición hará ganar a las escuelas -públicas y privadas- en eficiencia. Van a disputarse a los estudiantes y a intentar mejorar la calidad al mismo tiempo que disminuyen los gastos. $\mathrm{Al}$ animar a las escuelas privadas, los responsables van a permitir a los administradores de la educación desarrollar más innovación y, de este modo, mejorar los procesos de aprendizaje (Patrinos, 1999: 53). 
Toda la cuestión consiste, evidentemente, en aclarar precisar lo que se entiende por "calidad" y "mejorar". La calidad se entiende aquí, de manera exclusiva, como adecuación con las necesidades de la economía. Ahora bien, si pensamos la enseñanza como lugar de producción y de difusión de cultura, de valores y de saberes necesarios para la resolución de los grandes problemas de la humanidad, e incluso en el sentido mismo de la existencia (pobreza, desigualdades Norte-Sur, agotamiento de los recursos naturales, nivelación cultural, dictadura del relativismo, etc.), entonces la enseñanza superior mercantilizada promete catástrofes.

Nos encontramos frente a la inminencia de una dualidad social creciente de la enseñanza superior, con universidades de ricos y universidades de pobres en juego. Todavía con excesiva frecuencia, se dice también en los países en vías de desarrollo, que las universidades católicas son las de los ricos. Cuando las disparidades de financiación de las diferentes instituciones son demasiado grandes se vuelve cada vez más difícil garantizar una competición en pie de igualdad, incluso en los países ricos. En los Estados Unidos, por ejemplo, los costes crecientes en la enseñanza superior, añadidos a la reducción de los subsidios del Estado, han conducido a importantes disparidades en materia de recursos financieros entre las universidades públicas y privadas. De las veinte (20) primeras universidades americanas, solo dos (2): la Universidad de California (Berkeley) y la Universidad de Michigan, son universidades públicas. El foso creciente entre los salarios que se pagan en las universidades privadas y públicas constituye el elemento capital de esta evolución. Estas últimas experimentan a partir de ahí dificultades para atraer a los mejores profesores e investigadores.

Además de esta desigualdad, la mercantilización engendrará un estrecho repliegue de la universidad sobre los saberes que producen beneficios (rentables), a menudo en detrimento de las humanidades y de la libertad académica. La inseguridad, la visión reductora de la finalidad del conocimiento, las decisiones a corto plazo, la decadencia de la autonomía profesional individual, las presiones para realizar proyectos de investigación y para difundir los resultados de manera atractiva para los suministradores de fondos comerciales, se ocupan de socavar fuertemente la enseñanza superior, la universitas. 
¿Es ineludible todo eso? Los imperativos económicos en acción tienen ciertamente el aspecto de rodillo compresor, pero la historia no es lineal. La destrucción de la universitas, pública o privada, y de sus ambiciones, el empobrecimiento de los contenidos de la enseñanza, las condiciones de trabajo cada vez más difíciles, la precarización del estatuto de los trabajadores, los ataques contra la libertad académica: todo eso acaba por suscitar resistencias y luchas. La oposición a la mercantilización se desarrolla con la misma implacable necesidad que la misma mercantilización. El futuro de la enseñanza superior queda, en consecuencia, por escribir. Será fruto de estas fuerzas contrarias, de su enfrentamiento.

¿Hay alternativas? ¿Dónde están los universitarios en el momento en que se construyen las convergencias de resistencias al modelo económico, político y cultural dominante? ¿Estamos paralizados hasta tal punto por el sistema o disminuidos en nuestra dignidad de intelectuales? Existen alternativas: reforzar la universidad como servicio diversificado (público y también privado; confesional o no) abierto a todos, sacar la educación de la lógica mercantil, ofrecer espacios al pensamiento crítico, conectar la investigación con las necesidades prioritarias de la humanidad. ¿Quién tomará la iniciativa de constituir la universidad como agente social mundial portador de la defensa del sentido y de las necesidades esenciales a la condición humana? ¿Deberíamos elaborar una carta magna donde se reafirmen las bases de su misión científica y social, los principios de su identidad y de su funcionamiento y las perspectivas de su globalización? Me voy a tomar la libertad de soñar un instante ante ustedes: ¿podría ver la luz un foro mundial de las universidades, privadas y públicas, punto de encuentro de todos aquellos y aquellas que quieren reconstruir la función universitaria y ponerla al servicio del bienestar y de la igualdad de todos los seres humanos en todo el orbe planetario? ¿No sería ésta una de las tareas significativas de una Federación como la nuestra? ¿No tenemos aquí una razón más para continuar ahondando en nuestras reflexiones sobre los fenómenos de la mundialización/globalización?

\section{El desafío e la internalización vía "Internet"}

No se puede negar que uno de los más poderosos catalizadores de la transformación de la enseñanza superior en el gran mercado mundial es el desarrollo de las tecnologías de la comunicación a distancia y, en particular, el desarrollo 
de Internet. "El impacto de la tecnología sobre la educación es el motor del cambio", dice un informe del Banco Mundial. ${ }^{5}$ Así, tres grandes universidades americanas y una universidad inglesa (Columbia, Stanford, Chicago y London School of Economy) se han asociado en una compañía especializada en la difusión pedagógica vía Internet, a fin de crear la Cardean University que ofrece en todo el mundo, aunque solo por Internet, cursos de comercio, de administración y de contabilidad.

El Informe del Banco Mundial (2002) nos suministra toda una serie de informaciones. Una cuarta parte de los estudiantes que siguen cursos universitarios australianos desde el extranjero lo hacen mediante la enseñanza a distancia. En Canadá, el 57\% de las universidades proponen ya formaciones "en línea", que ofrecen más de 3.000 cursos. En Francia, la Université de Pau y la Université de Rennes en particular, ofrecen MBA o DESS de finanzas en la red Internet. En el año 2002, se estimaba que, solo los Estados Unidos, contaban con no menos de 3.000 instituciones especializadas en la enseñanza "en línea". Cabe destacar también que los países ricos no son, sin embargo, los únicos concernidos. La Universidad Virtual de Monterrey (México), propone 15 itinerarios de máster por vía de teleconferencias o de cursos virtuales. Éstos son seguidos por 50.000 estudiantes en los 1.450 centros de México y los 116 que existen en otras ciudades de América Latina. Por último, demos una tregua a las estadísticas, en Corea del Sur hay no menos de 15 universidades virtuales, que llegan a 14.500 estudiantes.

Al margen de la oferta de enseñanza superior propiamente dicha encontramos, asimismo en Internet, lo que recibe el nombre de "Academic Browsers": decenas de sociedades, como la Connect Education Inc. o la Electronic University Network, elaboran programas de enseñanza por ordenador, guían a los clientes (a menudo empresas), en sus necesidades de formación "en línea", sirven de intermediario entre universidades y estudiantes, proponen informaciones sobre los recursos académicos y financieros.

Podríamos sentir la tentación de alegrarnos. Es lo que vemos y oímos con frecuencia a nuestro alrededor. ¿Se harán accesibles, mañana, de este modo, los tesoros de la ciencia y de la cultura a la mayoría? ¿No nos estaremos olvidando

5 Banco Mundial, Constructing Knowledge Societies: New Challenges for Tertiary Education, Informe del Banco Mundial, 2002, p. 54. 
un poco rápido de que estos saberes no serán gratuitos de una manera duradera (ya es verdad que pocos de ellos lo siguen siendo) y que su acceso estará reservado, por tanto, a quien pueda pagarlos? Nos olvidaríamos, sobre todo, de que, como en toda globalización mercantil, una lucha a muerte traerá consigo la supervivencia de solo algunos. En los medios del Banco Mundial, ya es un dato claramente admitido que la eliminación de la barrera física resultante de la revolución de las nuevas tecnologías de la comunicación significa que, a partir de ahora, se hace posible que las universidades extranjeras compitan con las universidades locales y entren en contacto con estudiantes de todas partes, en todos los países. ${ }^{6}$

En este texto se plantea, por consiguiente, saber si eso no significará la muerte de las universidades locales, sobre todo en los países más pobres. Pues ¿cómo podrán competir con la oferta procedente de las prestigiosas instituciones americanas o europeas? Desde esta hipótesis, es la estandardización comercial y, por tanto, el empobrecimiento de la cultura (entre otras causas, por la dominación de una sola cultura y de una sola lengua) y del saber lo que nos espera al final del camino. Una vez más, en virtud de la fuerza del mercado, una tecnología potencialmente emancipadora se ve conducida a engendrar exactamente lo contrario de las promesas esperadas. También aquí se hunde en sus contradicciones el capitalismo en marcha.

Son también muchos los profesores de las universidades clásicas que no creen en la generalización de la enseñanza a distancia vía Internet. Dicen que la cosa no puede funcionar; estiman que el aliento, el alma de su vocación de educadores no se puede automatizar. “ ¡Ésa no será, hemos oído decir, más que una mala enseñanza!". Tal vez tengan razón en este punto. Pero la llevarán a cabo a pesar de todo, sean cuales fueren las consecuencias de para la calidad de la enseñanza. Seamos realistas, reconozcamos de manera perspicaz que lo que está en juego no es la educación, y menos aún el estudiante: jlo que está en juego es claramente el beneficio, el dinero!

Señoras y señores, queridos colegas y amigos, permítanme -antes de concluir- una última observación que afecta al conjunto de lo que he dicho hoy. Es 
justo y natural que el mundo universitario se interese por los desafíos y las interrogantes que plantea la posmodernidad, pues el objetivo de la universidad es ahondar, ir a la raíz de las cosas. Ella es el ámbito donde, en todos los tiempos, las diversas ramas del saber son objeto de investigación y de enseñanza; y la investigación y la enseñanza no pueden dejar de tener la verdad como punto de referencia. La verdad buscada, amada, enseñada y defendida, que es y debe ser como el alma de la universidad, porque ella es la vida profunda de la razón humana. El descubrimiento o el redescubrimiento de la magna tradición intelectual cristiana, del humanismo cristiano, puede constituir para nuestros medios un instrumento precioso para la reflexión sobre una visión del mundo y de la educación que esté plenamente a la altura del hombre y a la altura de Dios. Las cuestiones existenciales que afectan a la persona humana y a su destino, al mundo, encuentran un lugar escogido en los inmensos recursos del pensamiento bíblico, antropológico y filosófico cristiano. El nuevo humanismo, que constituye el preámbulo de la nueva evangelización, ¿no es el que escribiremos juntos, a ejemplo del Vaticano II, aceptando iniciar el diálogo con la posmodernidad, ese mundo que se construye sin Dios y, con excesiva frecuencia, sin el hombre?

La puesta en tela de juicio de la crisis de la universidad, tal como la podemos constatar en Europa y en otros lugares desde el final de la Segunda Guerra Mundial y a la que se intenta remediar, no es -en mi opinión- tanto de tipo organizativo como civilizacional, cultural y espiritual. No se trata tanto de una crisis de medios como de una crisis de identidad, de fines y de valores.

\section{Conclusión}

Queda claro que más allá del mundo de la educación, podemos observar también que las mutaciones científicas y tecnológicas, culturales, sociales y económicas son tan radicales en todos los campos que podemos imaginar $a$ priori tanto lo mejor como lo peor. El futuro está abierto, tanto en sentido positivo como negativo. Esto podemos ilustrarlo de múltiples maneras: en la economía, se puede producir un acceso de las mayorías a una civilización del ocio o, por el contrario, un paro masivo y pobreza; en la sociedad internacional se pueden forjar solidaridades más fuertes o, a la vez, también un repliegue nacionalista o proteccionista; en las ciencias biológicas y médicas se puede 
producir una mejora del servicio a la vida de los hombres y de las mujeres y a sus cuerpos o, en cambio, un deslizamiento hacia el aborto, la eugenesia, la eutanasia, el estallido de la célula familiar. Quisiera observar aquí que todos los grandes debates que han agitado la segunda mitad del siglo XX hasta hoy, y que probablemente lo seguirán haciendo, se reconocen en su manera de trastornar el sitio de la persona humana en la humanidad. Tengo la íntima convicción de que frente a tantos trastornos, la vocación de la universidad católica no es solo la de la ciencia, sino también la de la sabiduría. En nuestras sociedades actuales se impone como un deber prioritario de todos los educadores recentrar la persona humana, creada a imagen y semejanza de Dios.

Una sabiduría que proporciona a la universidad una voz crítica y profética sobre la sociedad y el mundo. En eso consiste su originalidad, ésa es su misión. Por eso, si se percibe hoy una urgencia en la vida de la universidad católica no es, a buen seguro, la de atenuar o difuminar su carácter cristiano, sino más bien ahondar en él, ponerlo de manifiesto, dar testimonio de él en el plano teórico y en el práctico. Debe tomar conciencia del incremento de las responsabilidades que le corresponden para verificar la autenticidad moral y humana de todos los nuevos desarrollos; la experiencia ha demostrado que el avance científico no equivale siempre, ni necesariamente, a un progreso moral y humano, equilibrado y compartido.

Esta misión indica no sólo un aumento del interés por la universidad católica, sino también una atención y una sensibilidad mayor por el valor eclesial de que está investida. La universidad católica está bien arraigada en la historia, en la sociedad y en la Iglesia. Una doble cuestión quedará planteada siempre e invito a los universitarios católicos a prolongar la re-flexión sobre ella: ¿Qué universidad católica hoy? La universidad católica: ¿Qué futuro? ¿Desafíos?, ¡ciertamente!, ¿Promesas? ¡aún más!

\section{Bibliografía}

Patrinos, H.A. "Market Forces in Education". Seminario sobre Educación: the Point of View of the Economists, Donostia-San Sebastián. España, 22-24 de julio de 1999. 